\title{
Underestimated Cervical Extradural Hematoma Secondary to the Small Needle-Scalpel for the Treatment of Cervical Spondylosis: A Rare but Avoidable Complication
}

\author{
Jinming Han, Meng-ge Yang, Qingxiang Zhang and Tao Jin* \\ Department of Neurology, Neuroscience Center, The First Hospital of Jilin University, Changchun, China
}

Objective: To present a case report highlighting a severe, yet avoidable, complication following small needle-scalpel treatment for cervical spondylosis.

Introduction: The small needle-scalpel is a miniature surgical instrument used to create intense and invasive punctures at certain acupoints with a small latch needle. It has been increasingly gaining popularity among clinicians and patients all over the world during the past years. However, severe complications after small needle-scalpel treatment have not

OPEN ACCESS

Edited by:

Irene Litvan,

University of California, San Diego,

United States

Reviewed by:

Paolo Missori,

Sapienza University of Rome, Italy

Roberto Colasanti,

Marche Polytechnic University, Italy

*Correspondence:

Tao Jin

drtao.jin@hotmail.com

Specialty section: This article was submitted to Spinal Cord Medicine, a section of the journal Frontiers in Neurology

Received: 30 December 2018 Accepted: 24 June 2019

Published: 05 July 2019

Citation:

Han J, Yang $M$, Zhang $Q$ and Jin T (2019) Underestimated Cervical

Extradural Hematoma Secondary to the Small Needle-Scalpel for the Treatment of Cervical Spondylosis: A

Rare but Avoidable Complication.

Front. Neurol. 10:740. doi: 10.3389/fneur.2019.00740 previously been reported.

Methods: Here we report a 54-year-old man who recently suffered from cervical spondylosis and underwent small needle-scalpel treatment, which was performed by a rural doctor. While there were no new neurologic deficits, the patient experienced delayed functional deterioration until the onset of quadriplegia within 1 month of treatment. Magnetic resonance imaging demonstrated a C2-C7 dorsally placed extradural hematoma with severe cord compression and subcutaneous soft tissue hemorrhage.

Results: The patient refused urgent corrective surgery and later died due to respiratory failure.

Conclusions: Although small needle-scalpel therapy has many benefits, such as reducing pain, shorter expenditure, shorter period of therapy and better recovery of function, there are also many potentially severe risks, such as cervical extradural bleeding, which requires clinicians to pay more attention to avoid the complications.

Keywords: cervical extradural hematoma, small needle-scalpel, MRI, complication, cervical spondylosis

\section{INTRODUCTION}

The small needle-scalpel is a miniature surgical instrument that creates intense and invasive punctures at certain acupoints with a small latch needle (1). The shape of the small needlescalpel looks like a needle with a sharp bladed tip and is $0.8 \mathrm{~mm}$ in diameter (Figure 1). Usually, manual manipulations, such as scratching between the skin layer and the fascia tissue, are necessary after the puncture is made, mostly to disconnect the parenchyma (Figure 2). It is sometimes regarded to as a part of the concept of acupuncture, but is more invasive than normal acupuncture. It was first introduced in clinics in China in 2000 for its therapeutic effect on disorders associated with muscle pain. A recent meta-analysis demonstrated that acupuncture has a 


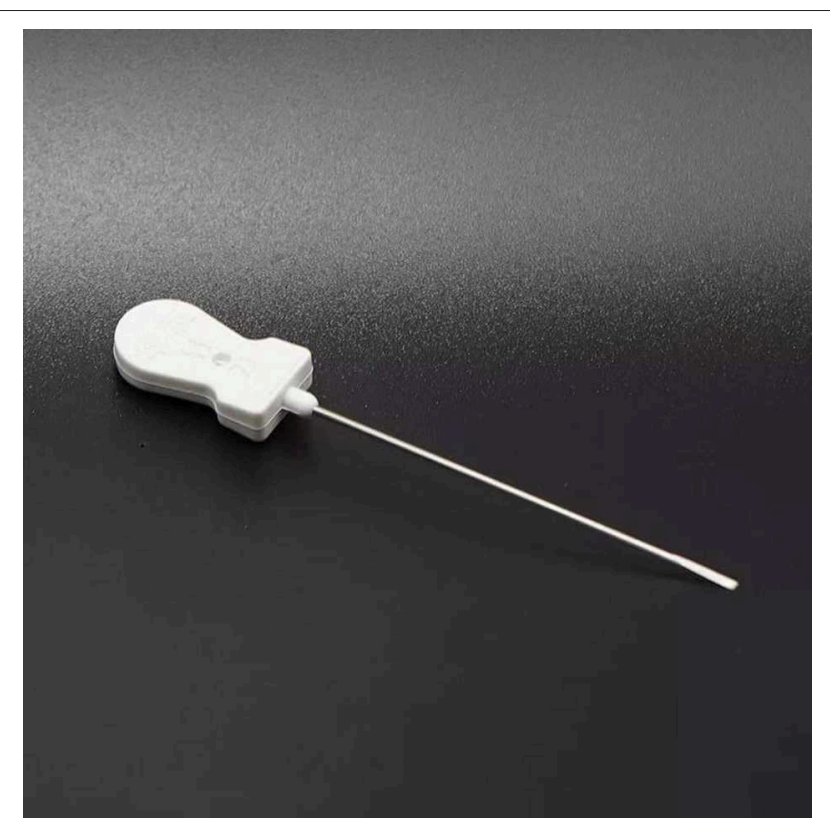

FIGURE 1 | Photograph of the small needle scalpel. The shape of the small needle-scalpel looks like a needle for acupuncture with a sharp bladed tip and $0.8 \mathrm{~mm}$ in diameter.

$33.41 \%$ effectiveness rate in the management of cervical spondylosis (2). It has increasingly gaining popularity among clinicians and patients throughout the world over the past years (3-6). A growing body of evidence also indicated that acupuncture may trigger a series of biological effects such as activating excitatory neurotransmission, promoting remyelination in the cuprizone-induced demyelinating animal model of multiple sclerosis, causing acupunctureinduced analgesia through purinergic signaling and reducing inflammatory response via the cholinergic anti-inflammatory pathway (7-10). Furthermore, this traditional technique has been considered an effective method for various forms of pain management including cervical spondylosis (2, 1113). Even though effective and safe, the acupoints for the treatment of cervical spondylosis were chosen according to the theory of traditional Chinese medicine and personal clinical experience based on relevant acupoints (Figure 3), which may vary from clinician to clinician $(14,15)$. Here we report a previously unrecognized complication of fatal cervical extradural hematoma secondary to the small needle-scalpel for the treatment of cervical spondylosis. This case report serves to raise the awareness of potential complications followed by this kind of treatment. Thus, comprehensive assessments before and after the small needle-scalpel treatment are of importance for the patient's prognosis.

\section{CASE PRESENTATION}

A 54-year-old Chinese man consulted a medical doctor with the complaint of recent painful neck and right upper limb without any clear causes. He had a medical history of left traumatic humeral fracture 10 years ago that did not significantly affect

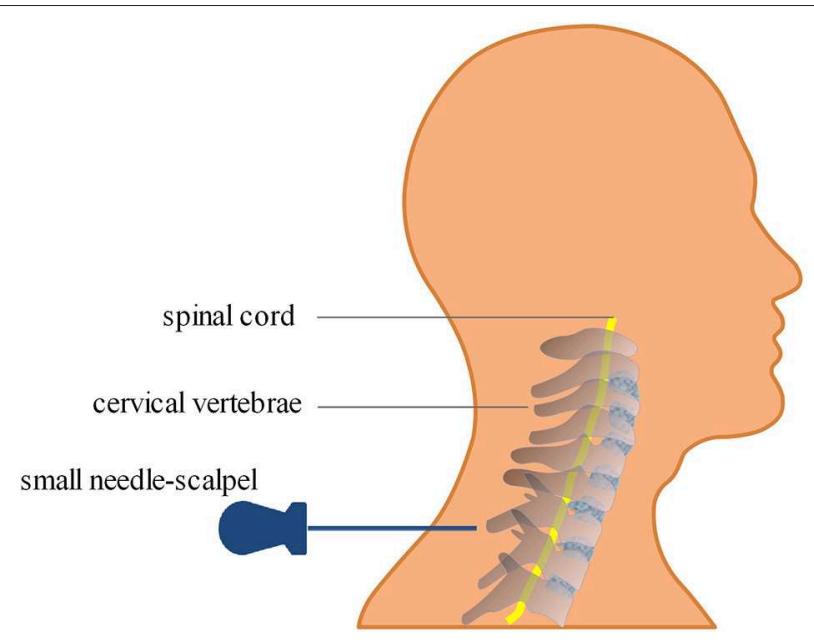

FIGURE 2 | Diagram of the small needle-scalpel treatment for cervical spondylosis. The small needle-scalpel is commonly used to punctured into specific sites within the subcutaneous layer of the neck, and then scratched between the skin layer and the fascia tissue after the puncture in order to disconnect the parenchyma.

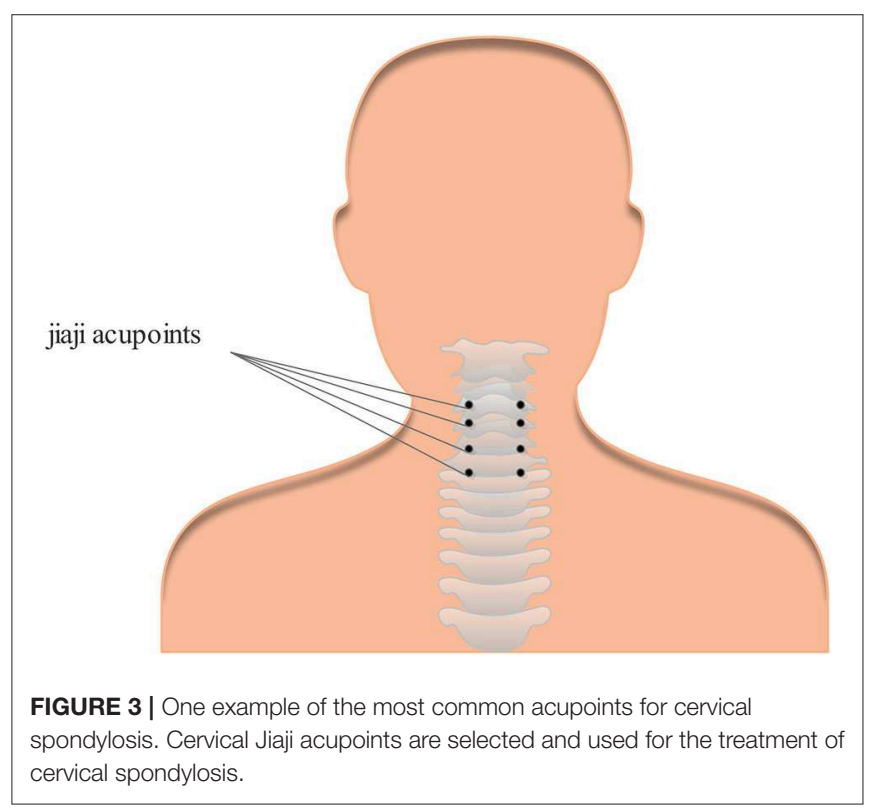

his recent daily life after surgery. The patient had no recent history of head or spinal trauma and did not take any related antiplatelet or anticoagulant drugs. In addition, the patient had no exposure to any toxic substances and no significant relevant family history. A clinical diagnosis of cervical spondylosis was originally considered based on X-rays of the cervical spine, and the patient received small needle-scalpel treatment for relieving pain relief, which was administered by a rural doctor. When no obvious improvement was observed 20 days later, the patient was admitted to our hospital for further diagnosis and treatment. The patient was experiencing progressive neurologic deterioration involving both upper and lower limbs. Upon neurological evaluation, he exhibited reduced response to light 


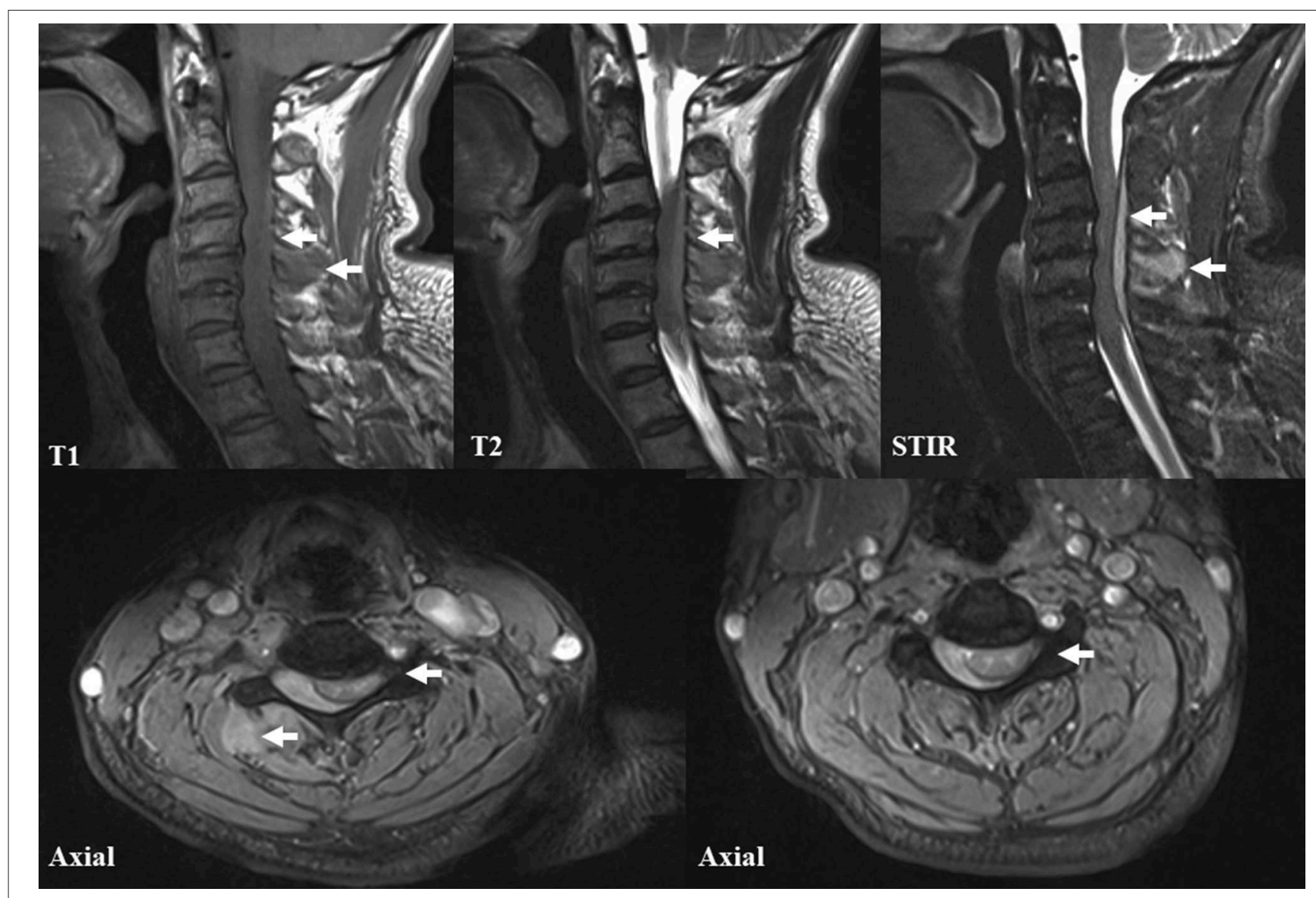

FIGURE 4 | Magnetic resonance imaging (MRI) of the cervical spine. The T1-weighted, T2-weighted, short tau inversion recovery (STIR) and axial cervical MRI images demonstrated a longitudinal dorsal extradural hematoma, extending from the $\mathrm{C} 2$ to $\mathrm{C} 7$ levels. In addition, severe cord compression and related subcutaneous soft tissue hemorrhage are also noted.

touch and pinprick, with a sensory level below the angle of the mandible. He had trouble during urination and significantly decreased muscle tension. He was subsequently incapable of antigravity power in his upper limbs (the left and right muscle strength was graded as $4 / 5$ and $2 / 5$, respectively) and had a significant loss of motor function in both lower extremities (the muscle strength was graded as 1/5). Urgent magnetic resonance imaging (MRI) of the cervical spine demonstrated a right spinal epidural hematoma located at the posterior spinal epidural space with severe cord compression and subcutaneous soft tissue hemorrhage, extending from the $\mathrm{C} 2$ to $\mathrm{C} 7$ spinal vertebral level (Figure 3). Routine laboratory investigations indicated that renal and liver functions, serum potassium and coagulation index were normal. Given his progressive symptoms and MRI results, the diagnosis of cervical extradural hematoma was finally considered. The patient had received the ventilatory and adjunctive therapy, omeprazole, mannitol, and a high dose of steroids after the diagnosis of cervical extradural hematoma. Unfortunately, the patient refused urgent surgery; thus, he continued to progressively deteriorate and later died due to respiratory failure.

\section{DISCUSSION}

Cervical extradural hematoma is an uncommon but highly disabling neurologic emergency in clinical practice that causes morbidity without early surgical intervention (16). The etiology of cervical extradural hematoma may be due to spontaneous and traumatic factors (16-18). Patients with cervical extradural hematoma typically present with sudden severe neck pain followed by rapidly progressive symptoms (19). Furthermore, the initial clinical signs and manifestations of cervical extradural hematoma are usually atypical, presenting unspecific clinical symptoms and mimicking other diseases that make this disease more difficult to recognize (17). However, if clinicians fail to recognize this disease early, it may lead to catastrophic consequences for patients. Here we report an underestimated, but serious case of cervical extradural hematoma that occurred secondary to small needle-scalpel for the treatment of cervical spondylosis.

Although widely considered effective and safe, several potential side effects, such as neural tissue injury, skin infection, and bleeding adhesion, have been reported with the use of 
acupuncture, with having a $8.6 \%$ incidence of side effects $(1,19,20)$. However, secondary cervical extradural hematoma after small needle-scalpel treatment has not yet been reported. Initially diagnosed with cervical spondylosis, the patient reported here received the small needle-scalpel treatment to relieve pain while being treated by a rural medical doctor. The cause of bleeding related to the cervical extradural hematoma can be both venous and arterial in origin. Specifically, the small needle-scalpel involved the insertion of fine needles into specific sites on the body within the subcutaneous layer. Manual manipulations such as scratching between the skin layer and the fascia tissue are needed after the puncture, mostly to disconnect the parenchyma (Figure 2). In our patient, related subcutaneous soft tissue hemorrhage were obvious on MRI scans (Figure 4). In addition, this patient had no recent history of head or spinal trauma and was not taking related antiplatelet or anticoagulant drugs. Thus, the causal relationship between cervical extradural hematoma and small needle-scalpel treatment can be rated as highly probable. Small needle-scalpel treatment has the potential risk to cause fatal extradural bleeding; however, this risk is largely ignored by clinicians. In our patient, the small needle-scalpel surgery was performed by a rural medical doctor who may not have had sufficient experiences. The acupoints that were chosen for treatment vary between clinicians and inappropriate operation may damage the extradural blood vessel and cause extradural bleeding risk. In a previous study, an 47-year-old Indian patient was reported to have immediately suffered from a cervical extradural hematoma when reversed from anesthesia after spinal surgery that was noted in the posterior cervicomedullary junction and lead to a compression of the cervical cord (16). However, in our case, the patient gradually experienced progressive neurologic deterioration within 1 month after small needle-scalpel treatment without any other recent traumatic evidence. Therefore, the possibility of delayed emergence

\section{REFERENCES}

1. Lin W, Liu CY, Tang CL, Hsu CH. Acupuncture and small needle scalpel therapy in the treatment of calcifying tendonitis of the gluteus medius: a case report. Acupunct Med. (2012) 30:142-3. doi: 10.1136/acupmed-2012-010149

2. Deng YZ, Xu LG, Chen L, Zhou D, Liu Y. Effectiveness of acupuncture in the management of cervical spondylosis: a meta-analysis. J Biol Regul Homeost Agents. (2017) 31:1017-22.

3. Han JS, Ho YS. Global trends and performances of acupuncture research. Neurosci Biobehav Rev. (2011) 35:680-7. doi: 10.1016/j.neubiorev.2010.08.006

4. Liu S, Zhang CS, Cai Y, Guo X, Zhang AL, Xue CC, et al. Acupuncture for post-stroke shoulder-hand syndrome: a systematic review and meta-analysis. Front Neurol. (2019) 10:433. 10:433. doi: 10.3389/fneur.2019.00433

5. Williams H, Sweet L, Graham K. Acupuncture during pregnancy and the perinatal period: women's attitudes, beliefs and practices. Women Birth. (2019). doi: 10.1016/j.wombi.2019.04.010. [Epub ahead of print].

6. Lee SH, Lee J, Lee YJ, Kim MR, Cho JH, Kim KW, et al. Effectiveness and cost-effectiveness of acupuncture with Doin therapy for chronic neck pain: a study protocol for a multicentre, randomised controlled clinical trial. BMJ Open. (2019) 9:e026632. 9:e026632. doi: 10.1136/bmjopen-2018-026632

7. Zhu K, Sun J, Kang Z, Zou Z, Wu G, Wang J. Electroacupuncture promotes remyelination after cuprizone treatment by enhancing myelin following small needle-scalpel treatment must be considered by clinical neurologists.

In conclusion, although there are many benefits, such as reducing pain, less expenditure, a shorter period of therapy, and better recovery of function, small needle-scalpel treatment may also have the potential to cause severe risks, such as cervical extradural bleeding which requires clinicians to pay more attention to avoid related complications. Precisely choosing the acupoints, standardized surgery and long-term follow-up may avoid related complications followed by small needlescalpel treatment.

\section{ETHICS STATEMENT}

As this is a case report without experimental intervention, no formal research ethics approval is required. The relatives of this patient provided written informed consent to the publication of the information and images related to this case report.

\section{AUTHOR CONTRIBUTIONS}

JH drafted the manuscript. MY and QZ helped to prepare the figures and collected data. TJ performed a critical revision.

\section{FUNDING}

This work was supported by grants from the General Program of the National Natural Science Foundation of China (No. 81671177), the Natural Science Foundation of Jilin Province Science and Technology Development Plan Project (20190201043JC), the Technology Innovation Program of Jilin Provincial Health and Family Planning Commission of China (No. 2016J040), as well as grants from the First Hospital, Jilin University of China. debris clearance. Front Neurosci. (2016) 10:613. 10:613. doi: 10.3389/fnins. 2016.00613

8. Zhang XF, Xiang SY, Geng WY, Cong WJ, Lu J, Jiang CW, et al. Electroacupuncture regulates the cholinergic anti-inflammatory pathway in a rat model of chronic obstructive pulmonary disease. J Integr Med. (2018) 16:418-26. doi: 10.1016/j.joim.2018.10.003

9. Chen HC, Chen MY, Hsieh CL, Wu SY, Hsu HC, Lin YW. TRPV1 is a responding channel for acupuncture manipulation in mice peripheral and central nerve system. Cell Physiol Biochem. (2018) 49:1813-24. 49:1813-24. doi: 10.1159/000493627

10. Tang Y, Yin HY, Liu J, Rubini P, Illes P. P2X receptors and acupuncture analgesia. Brain Res Bull. (2018). doi: 10.1016/j.brainresbull.2018.10.015. [Epub ahead of print].

11. Garner BK, Hopkinson SG, Ketz AK, Landis CA, Trego LL. Auricular acupuncture for chronic pain and insomnia: a randomized clinical trial. Med Acupunct. (2018) 30:262-72. 30:262-72. doi: 10.1089/acu. 2018.1294

12. Sung SH, Sung AD, Sung HK, An TE, Kim KH, Park JK. Acupuncture treatment for chronic pelvic pain in women: a systematic review and meta-analysis of randomized controlled trials. Evid Based Complement Altern Med eCAM. (2018) 2018:9415897. doi: 10.1155/2018/94 15897 
13. Jiang $Y$, Bai $P$, Chen $H$, Zhang XY, Tang XY, Chen HQ, et al. The effect of acupuncture on the quality of life in patients with migraine: a systematic review and meta-analysis. Front Pharmacol. (2018) 9:1190. doi: 10.3389/fphar.2018.01190

14. Ma SX. Nitric oxide signaling molecules in acupoints: toward mechanisms of acupuncture. Chin J Integr Med. (2017) 23:812-5. doi: 10.1007/s11655-017-2789-x

15. Sun ZR, Yue JH, Zhang QH. Electroacupuncture at Jing-jiaji points for neck pain caused by cervical spondylosis: a study protocol for a randomized controlled pilot trial. Trials. (2013) 14:360. doi: 10.1186/1745-6215-14-360

16. Tomar GS, Kumar S, Dube SK, Goyal K. Postoperative extradural hematoma of the cervical spine: a rare but avoidable complication. J Clin Anesth. (2016) 31:120-1. 31:120-1. doi: 10.1016/j.jclinane.2016.02.019

17. Li C, He R, Li X, Zhong Y, Ling L, Li F. Spontaneous spinal epidural hematoma mimicking transient ischemic attack: a case report. Medicine (Baltimore). (2017) 96:e9007. doi: 10.1097/MD.0000000000009007

18. Kondo A, Yamaguchi H, Ishida Y, Toyoshima D, Azumi M, Akutsu N, et al. Spontaneous spinal epidural hematoma mimicking Guillain-Barre Syndrome. Brain Dev. (2019) 41:392-5. doi: 10.1016/j.braindev.2019.05.005
19. Eghbal K, Ghaffarpasand F. An acute cervical subdural hematoma as the complication of acupuncture: case report and literature review. World Neurosurg. (2016) 95:616 e11-3. doi: 10.1016/j.wneu.2016. 08.090

20. Ji GY, Oh CH, Choi WS, Lee JB. Three cases of hemiplegia after cervical paraspinal muscle needling. Spine J. (2015) 15:e9-13. doi: 10.1016/j.spinee.2014.11.007

Conflict of Interest Statement: The authors declare that the research was conducted in the absence of any commercial or financial relationships that could be construed as a potential conflict of interest.

Copyright (c) 2019 Han, Yang, Zhang and Jin. This is an open-access article distributed under the terms of the Creative Commons Attribution License (CC BY). The use, distribution or reproduction in other forums is permitted, provided the original author(s) and the copyright owner(s) are credited and that the original publication in this journal is cited, in accordance with accepted academic practice. No use, distribution or reproduction is permitted which does not comply with these terms. 